\title{
Dravet Syndrome: Diagnosis and Long-Term Course
}

\author{
Mary B. Connolly
}

\begin{abstract}
Dravet syndrome is one of the most severe epilepsy syndromes of early childhood, and it comes with very high morbidity and mortality. The typical presentation is characterized by hemiclonic or generalized clonic seizures triggered by fever during the first year of life, followed by myoclonic, absence, focal and generalized tonic-clonic seizures. Non-convulsive status epilepticus and epileptic encephalopathy are common. Development is normal in the first year of life, but most individuals eventually suffer from intellectual impairment. Dravet syndrome is associated with mutations in the sodium channel alpha1 subunit gene (SCN1A) in 70-80\% of individuals. SCN1A mutation results in inhibition of the GABAergic inhibitory interneurons, leading to excessive neuronal excitation. The "interneuron hypothesis" is the current most accepted pathophysiological mechanism of Dravet syndrome. The mortality rate is increased significantly in Dravet syndrome. Ataxia, a characteristic crouched gait and Parkinson's symptoms may develop in some individuals. It is likely that Dravet syndrome is underdiagnosed in adults with treatment-resistant epilepsy. Early diagnosis is important to avoid anti-seizure medications that exacerbate seizures.
\end{abstract}

Keywords: Dravet syndrome, electroclinical features, comorbidities, prognosis

doi:10.1017/cjn.2016.243

Can J Neurol Sci. 2016; 43: S3-S8

\section{INTRODUCTION}

Dravet syndrome (previously known as "severe myoclonic epilepsy of infancy") was first described by Dr. Charlotte Dravet in $1978 .{ }^{1}$ It is an early-onset treatment-resistant epilepsy syndrome that typically presents during the first year of life. The incidence of Dravet syndrome is estimated at 1 per 22,000-40,000 based on studies in the United Kingdom and Denmark, ${ }^{2,3}$ and it affects males twice as often as females. It typically causes an epileptic encephalopathy. In 2001, Claes and colleagues ${ }^{4}$ discovered mutations in the sodium channel alphal subunit gene SCN1A in seven individuals with Dravet syndrome. SCN1A mutations, usually de novo, are found in 70 to $80 \%$ of patients with Dravet syndrome. It is now appreciated that the electroclinical features of Dravet syndrome are broader than the original description and that the myoclonic seizures or generalized spike and wave are not present in all patients. Focal seizures that may evolve to generalized convulsive seizures are the dominant seizure type in some individuals and are associated with multifocal EEG abnormalities. With the wider application of new genomic technologies in the diagnosis of epilepsy of unknown cause, it is recognized that other genetic abnormalities ( $P C D H 19$, $G A B R G 2, S C N 1 B$ and $S C N 2 A$ ) may cause a similar phenotype as Dravet syndrome.

\section{Clinical Presentation and Evolution of Epilepsy in DraVET SYNDROME}

Seizure onset is typically in the first year of life, with prolonged, febrile and afebrile hemiclonic or generalized clonic seizures in previously healthy children. They may be associated with vaccinations or hyperthermia, including a warm bath. Over the subsequent months, affected individuals experience recurrent febrile and afebrile seizures that often affect alternate sides of the body. Between one and four years of age, other seizure types develop, including myoclonic and atypical absences, focal seizures and generalized tonic-clonic seizures. Focal seizures, with or without impairment of awareness, may be associated with such prominent autonomic features as pallor, cyanosis and drooling, and may evolve into a focal motor or bilateral convulsive seizure. Tonic seizures are reported to be uncommon in Dravet syndrome. In some individuals, myoclonic seizures do not develop and other seizure types, particularly focal or multifocal seizures, are the predominant seizure types.

In a retrospective survey ${ }^{5}$ of 138 children with Dravet syndrome with SCN1A mutations from China, seizure onset was before the age of 7 months in $77 \%$. $72 \%$ of the children in that study had febrile seizures with a duration longer than 15 minutes, and $67 \%$ had two or more febrile seizures within a 24-hour period. Seizures were hemiclonic with fever in $80 \%{ }^{5}$ In a study of 96 children who had febrile seizures prior to one year of age, 46 of whom had Dravet syndrome and 50 of whom did not, the factors that correlated significantly with Dravet syndrome were onset of febrile seizures under the age of 7 months, 5 or more seizures, duration of seizures longer than 10 minutes, hemiconvulsions, focal seizures, myoclonic seizures and hot-water-induced seizures. ${ }^{6}$ Reflex seizures are frequent, and the most common trigger is hyperthermia

\footnotetext{
From the Department of Paediatrics, British Columbia's Children's Hospital and University of British Columbia, Vancouver, British Columbia, Canada.

Received February 11, 2016. Final Revisions Submitted April 19, 2016. Date of ACCEPTANCE May 26, 2016.

Correspondence to: Mary Connolly, Clinical Professor of Paediatrics (Neurology),

Head, Division of Paediatric Neurology, and Director of the Epilepsy Program, British

Columbia's Children's Hospital, 4480 Oak Street, Vancouver, British Columbia

V6H 3V4, Canada. Email: mconnolly@cw.bc.ca.
} 
(fever, immersion in hot water, intense physical exercise or high ambient temperature).

Status epilepticus occurs commonly in Dravet syndrome, both the convulsive and non-convulsive types. Non-convulsive status epilepticus is also described in the literature as "obtundation status epilepticus." Obtundation status epilepticus is characterized by altered awareness, autonomic symptoms and subtle myoclonus that commonly involves the fingers and orobuccal muscles, and it may last for many hours or days. ${ }^{7}$ Status epilepticus may be life threatening, and the symptoms and signs of non-convulsive status epilepticus may be subtle and difficult to appreciate. Thus, it is of utmost importance to educate parents and caregivers about the early symptoms and signs of non-convulsive status epilepticus. It is also very important to put an individualized emergency (rescue) management plan in place for each individual so as to facilitate early intervention to prevent prolonged status epilepticus and its associated complications.

With respect to the evolution of epilepsy over time, seizures tend to become less frequent and severe in adolescence and adulthood. Fever sensitivity persists but has less impact. Myoclonic, atypical absence and focal seizures with altered awareness are less common in adulthood. ${ }^{8}$ The most common seizure type in adulthood is generalized tonic-clonic, which may be focal in onset and occurs mainly during sleep. Patients may also experience bilateral or asymmetrical posturing, which may be followed by tonic vibratory or clonic movements. However, there are few studies that describe in detail the electroclinical features of the seizure types in Dravet syndrome during adolescence or adulthood. Ictal recordings of five adults revealed subclinical focal seizures in three, focal seizures that evolved into bilateral convulsive seizures (secondarily generalized tonic-clonic seizures) in three, obtundation status in one and tonic events in another patient. ${ }^{8}$ In a long-term study ${ }^{9}$ of 53 children aged 4 to 14 years who were followed for from 3 to 14 years, seizure frequency was weekly in $74 \%$, monthly in $13 \%$ and daily in $13 \%$. The most common treatments employed in this study were valproic acid, clobazam and a ketogenic diet.

Takayama and colleagues ${ }^{10}$ described 64 patients followed at one epilepsy center in Japan. The median age at last follow-up was 30 years (range $=19-45$ ), and only 1 of 44 individuals with typical Dravet syndrome was in remission, in contrast with 4 of 20 individuals with atypical Dravet syndrome. In this study, atypical Dravet syndrome was characterized by absence of myoclonic and atypical absence seizures.

\section{EEG Evolution in Dravet Syndrome}

The EEG is typically normal during the first year of life. Subsequently, the EEG background may be normal or slow. Sleep architecture is typically preserved. Generalized spike and wave, polyspike wave and multifocal spikes are common.

Few studies have carefully documented the evolution of EEG findings over time in Dravet syndrome. Speechio and colleagues ${ }^{11}$ described EEG findings in 22 children with Dravet syndrome over the first five years following diagnosis. In all these children, the EEG background was normal, but background slowing was seen in $27 \%$ after six months. Epileptiform discharges were present in $27 \%$ at seizure onset and in $64 \%$ by 5 years follow-up. Multifocal epileptiform discharges were observed in $57 \%$, focal epileptiform discharges in $29 \%$, and $14 \%$ had generalized epileptiform discharges. A photoparoxysmal response was seen in $9 \%$ at seizure onset and in $41 \%$ by the end of the study. In a study of 16 children, Korff et al. ${ }^{12}$ reported that the EEG was abnormal in $81 \%$. However, at the time of seizure onset, the EEG was abnormal in only $25 \%$ of the children. The most common epileptiform pattern consisted of generalized spike wave discharges. ${ }^{12}$

In a study of 23 patients in whom seizures were recorded during video-EEG monitoring, Kim and colleagues ${ }^{13}$ divided patients into three groups: 0-5 years $(n=7), 6-10$ years $(n=11)$ and 11 years and older $(n=5)$. Slowing of the EEG background was more common in older patients and a photoparoxysmal response in younger children. Epileptiform discharges were found in most patients, and the most common pattern was multifocal epileptiform discharges.

Nabbout et al. ${ }^{14}$ also described five adolescents with Dravet syndrome who had bifrontal spike and slow wave and generalized fast polyspikes in sleep, features similar to Lennox-Gastaut syndrome. In another study of EEG evolution in 24 patients with Dravet syndrome, Genton et al. ${ }^{8}$ described normal EEG background in 8 and slow and disorganized background in 11 . Epileptiform activity was multifocal in 11, focal in 7 and generalized in 6 . Photosensitivity and pattern sensitivity tended to disappear by 20 years of age.

There is still an opportunity to add to the literature on the evolution of the electroclinical features of Dravet syndrome with SCN1A mutations over time, and additional ictal EEG data would be very informative.

\section{FAmily History of EPILEPSy/Febrile SeIZUReS}

The first report of SCN1A mutations in epilepsy was in the syndrome of genetic epilepsy with febrile seizures plus. ${ }^{15} \mathrm{~A}$ family history of epilepsy or febrile seizures has been observed in $15-35 \%$ of individuals with Dravet syndrome, and the most common phenotype seen in affected relatives is genetic epilepsy with febrile seizures plus. ${ }^{16}$ Dravet syndrome has been reported in monozygotic twins and rarely in zygotic twins. There are also rare reports of two or more affected children in the same family. Somatic or germline mosaic mutations may explain an unaffected or mildly affected parent, and in one study ${ }^{17}$ mosaicism was found in $7 \%$ of families with Dravet syndrome. The observation of a positive family history of febrile seizures and epilepsy in individuals with Dravet syndrome and de novo mutations in SCN1A suggest that the mode of inheritance is polygenic and that other modifier genes such as $S C N 9 A$ contribute to the phenotype.

\section{Genetic Testing in Dravet Syndrome}

The first mutations in the SCN1A gene in seven individuals with Dravet syndrome were discovered by Claes and colleagues ${ }^{4}$ in 2001. It is now recognized that $S C N 1 A$ mutations are seen in $75-80 \%$ of individuals with Dravet syndrome. More than 500 mutations have been reported in individuals with Dravet syndrome. Mutations are truncating in 40-50\%, missense in $40 \%$ and splice site in the remainder. Whole-gene deletions are observed in 2-3\%, and gene duplications are rare. Most gene mutations are de novo, but familial mutations occur in $5-10 \%$ and are usually missense. $^{4,18,19}$

Truncating, nonsense and frameshift mutations and gene deletions correlate with the classical Dravet syndrome phenotype with an earlier age at onset. ${ }^{20,21}$ Missense mutations that affect 


\section{Table 1: Predominant seizure types in Dravet syndrome}

\begin{tabular}{l}
\hline Children: \\
\hline Hemiclonic or generalized clonic seizures \\
\hline Focal seizures ( \pm evolution to generalized tonic-clonic seizures) \\
\hline Atypical absence seizures \\
\hline Myoclonic seizures \\
\hline Adults: \\
\hline Focal seizures \pm evolution to generalized tonic-clonic seizures
\end{tabular}

the pore-forming region are associated with a more severe phenotype. Individuals who test negative for SCN1A sequencebased mutations may have $S C N 1 A$ exon deletions or chromosomal rearrangements involving $S C N 1 A$ and contiguous genes, which may be detected using multiplex ligation-dependent probe amplification (MPLA) and comparative genome hybridization (CGH). The Genetics Commission of the International League Against Epilepsy (ILAE) has recently published guidelines for SCN1A testing in clinical practice. ${ }^{22}$ Their recommendations are summarized in Table 1.

The phenotype of SCN1A mutations may be heterogeneous even within the same family, and it is likely that modifying genes are important in determining the severity of epilepsy and possibly comorbidities. ${ }^{2}$ In animal models, SCN8A may restore a normal seizure threshold in the presence of $S C N 1 A$ mutations. ${ }^{23}$ The compensatory up-regulation of $S C N 3 A$ may mitigate the effects of Nav1.1 deficiency. Variants in CACNA1A genes may also modify the phenotype in Dravet syndrome. In a study ${ }^{24}$ of 48 patients with Dravet syndrome and SCN1A mutations, $C A C N A 1 A$ variants were observed in 20 subjects. In individuals with $C A C N A 1 A$ variants, absence seizures, earlier age at seizure onset and more frequent prolonged seizures before the age of one year were more common than in Individuals without CACNA1A variants. ${ }^{24} S C N 9 A$ mutations have also been observed in some individuals with $S C N 1 A$ mutations and may be associated with a more severe clinical phenotype. ${ }^{25,26}$

In children where $S C N 1 A$ mutations are not found, several other genes have been reported, including CHD2, GABRA1, GABARG2, STXBP1, SCN1B, SCN2A and PCDH19. ${ }^{27-29}$ However, the electroclinical phenotypes associated with these other gene abnormalities are often somewhat atypical for Dravet syndrome. With the wider application of next-generation sequencing in the evaluation of epilepsy of unknown cause, it is likely that the electroclinical features associated with gene mutations other than SCN1A will be better described.

\section{Pathophysiology of Dravet Syndrome}

Several pathophysiological mechanisms have been reported in Dravet syndrome, and the most accepted is the "interneuron hypothesis," where SCN1A mutations result in inhibition of the GABAergic inhibitory interneurons, resulting in excessive excitation. This mechanism is supported by a mouse model. ${ }^{30}$ Nav1.1 was preferentially expressed in the axon initial segments of the parvoalbumin-positive interneurons, the main type affected in Dravet syndrome. ${ }^{31}$ Studies using models involving humanderived induced pluripotent stem cell neurons suggest that both
GABAergic inhibitory neurons and glutamatergic excitatory neurons in the forebrain of patients with Dravet syndrome are hyperexcitable with increased sodium current density, overall resulting in network hyperexcitability. ${ }^{32}$ SCN1A haploinsufficiency may result in a compensatory increase in the sodium current via expression of other sodium channels. ${ }^{33}$

\section{Neuroimaging in Dravet Syndrome}

Structural brain imaging using MRI is usually normal in Dravet syndrome, but imaging abnormalities are described in some patients. In a review ${ }^{34}$ of 120 patients in Italy with Dravet syndrome, malformations of cortical development were detected in 4 . In another study, ${ }^{35}$ of 18 children with Dravet syndrome who had MR imaging after 3 years of age, 7 had hippocampal sclerosis or loss of grey-white definition in the temporal lobe. In a study ${ }^{36}$ comparing nine patients with Dravet syndrome and SCN1A mutations and nine controls without seizures, globally reduced grey and white matter volumes were seen in individuals with Dravet syndrome.

\section{NeurodeVelopment in Dravet SyNdrome}

Neurodevelopment and formal neurological examination are typically normal at the time of seizure onset. However, there is slowing of the rate of the developmental progress along with variable decline in the developmental quotient over time. ${ }^{37}$ Ataxia and pyramidal signs were present in 59 and $22 \%$ of subjects, respectively. ${ }^{37}$ Attention, visual/motor integration, visual perception and executive function are impaired, whereas language may be less involved in some patients. ${ }^{38,39}$

In a study ${ }^{40}$ of 21 children aged 6 to 10 years with Dravet syndrome, seen at a single institution and assessed using the WISC and Vineland Adaptive Behaviour scales, no child had a normal IQ after the age of 6 years. Only 5 of the 21 children had a verbal or non-verbal IQ score greater than 60 . The children had attention problems, impulsivity, perseverative responses and deficits in planning. Socialization skills assessed using the Vineland scale were significantly better than communication skills. No correlation was observed between age at seizure onset, status epilepticus, number of seizures, myoclonic or absence seizures with IQ. Nabbout and colleagues ${ }^{41}$ reported cognitive findings in 67 children with Dravet syndrome, 52 of whom were tested only once and 15 underwent repeat testing. IQ was typically normal before the age of 2 years (mean of 80) but low after age 3 years (mean $=48$, range $=30-69$ ). However, there was no evidence of regression. Attention and hyperactivity were common. No significant correlation was seen between IQ and age at first seizure, number of episodes of status epilepticus, or myoclonic and focal seizures. However, IQ was lower in those with SCN1A mutations. Thus, it was the conclusion of the authors that the encephalopathy was not a pure consequence of epilepsy but that the SCN1A mutation played an additional direct role.

Genton and colleagues ${ }^{8}$ reported 24 patients with Dravet syndrome followed to 20-50 years of age. Only three individuals lived independently, and the remainder had moderate to severe delay. Ataxia was seen in nine, dysarthria in eight and tremor in seven. In another report of 31 patients, only 1 lived independently, 7 had no language and 9 spoke only a few words. ${ }^{42}$ Catarino et al. ${ }^{43}$ described the long-term follow-up in 22 adults followed to 


\section{Table 2: ILAE guidelines for $S C N 1 A$ testing ${ }^{22}$}

1. SCN1A testing should be performed in cases of suspected Dravet syndrome and in some other early-onset infantile epileptic encephalopathies. Dravet syndrome

should be considered in infants presenting around six months of age, with prolonged and recurrent, febrile, hemiclonic seizures, or seizures induced by bathing. Genetic confirmation of clinical diagnosis may allow optimization of AED therapy with improved seizure control and developmental outcome, may prevent unnecessary investigations and may inform genetic counselling.

2. SCN1A testing could be considered in developmentally normal infants with recurrent febrile or afebrile, prolonged, hemiclonic seizures or generalized status epilepticus.

3. SCN1A testing is not recommended for children with phenotypes not suggestive of SCN1A mutations such as abnormal development or neurological deficits present at birth, or structural abnormalities of the brain.

a median of 39 years. Most had intellectual disability, 16 lived in residential care and 6 at home with support. Takayama and colleagues $^{10}$ reported on long-term outcome in 64 patients followed to a median of 30 years. All had intellectual disability, which was severe in $75 \%$, moderate in $20 \%$ and mild in $5 \%$. Only two individuals lived independently.

In Dravet syndrome, there is widespread dysfunction of Nav1.1. Maturation of sodium channels in humans and mice shows that Nav1.1 expression is very low in neonates, and other alpha subunits Nav1.2 and 1.3 compensate. Normally, with increasing age, Nav1.3 declines and Nav1.1 increases. In Dravet syndrome, Nav1.1 fails to increase normally, resulting in widespread dysfunction of Nav1.1. This may explain why seizures do not start until later on, during the first year of life. Furthermore, mouse models ${ }^{44}$ of Dravet syndrome demonstrate hyperactivity, stereotypic behaviour and cognitive deficits due to reduced expression of GABAergic interneurons in the forebrain. In a study $^{45}$ of mice where $S C N 1 A$ was selectively reduced but the animals did not develop seizures, cognitive function was affected, providing further evidence that Nav1.1 dysfunction plays a role independent of seizures on cognitive decline.

\section{Comorbidities in Dravet Syndrome}

A crouch gait has been described in many reports on Dravet syndrome. In one study ${ }^{46}$ utilizing formal gait analysis in patients with Dravet syndrome who ranged in age from 2 to 34 years, crouch gait was seen in 8 of 9 children aged 13 years and older, in $50 \%$ of children aged 6-12 years and in no child younger than 6 years. Fasano and colleagues, ${ }^{47}$ in a study of 12 adults with Dravet syndrome, described features of Parkinsonism with bradykinesia, asymmetric rigidity and cogwheeling in $11(91 \%)$. Two patients were treated successfully with levodopa, resulting in significant improvement in their Parkinsonian symptoms. Osteopenia and increased risk of fractures are common in individuals with treatment-resistant epilepsy, particularly when enzyme-inducing drugs are used or there is reduced mobility, but this has not been studied in Dravet syndrome.

Despite sleep concerns being a common complaint in individuals with Dravet syndrome, the literature on this topic is limited. A small, retrospective study ${ }^{48}$ of polysomnogram findings in six children with Dravet syndrome referred for sleep assessment found no abnormalities in sleep macroarchitecture despite parental concerns.

\section{SUDEP and Mortality}

Mortality is increased significantly in Dravet syndrome, and death may occur at any age but is more frequent during childhood. Death may be due to status epilepticus, SUDEP or accidental death, and it may also be related to seizures associated with drowning or injury. Mortality rates have varied in reported studies from 3.1 to $20.8 \%$, with deaths due to sudden, unexpected death of someone with epilepsy (SUDEP), status epilepticus and accidents (especially drowning) being the most common. In a report ${ }^{49}$ based on data from a patient support group, it was estimated that individuals with Dravet syndrome have a risk of SUDEP approximately 15 -fold higher than those with other childhood epilepsies.

Studies in Dravet mice models ${ }^{50}$ suggest that SUDEP is due to increased parasympathetic activity after a generalized tonic-clonic seizure, resulting in lethal bradycardia and electrical ventricular dysfunction. As this finding was seen in mice with the SCN1A mutations selectively targeted to affect the brain, but not if the SCN1A mutations were selectively targeted to affect the heart only, it was proposed that SUDEP is due to a brain effect on the heart after a seizure. Cheah and colleagues ${ }^{51}$ proposed that the natural decline in Nav1.3 channel expression with the failure of increase in Nav1.1 channel expression leads to disinhibition of neuronal circuits, treatment-resistant epilepsy and premature death. In a study $^{52}$ of mice with an SCN1A mutation, Auerbach observed a twofold increase in sodium current density in ventricular myocytes, resulting in increased excitability, prolongation of action potential duration, which results in QT prolongation, abnormal rhythms and spontaneous deaths in some mice. Thus, it is possible that altered electrical cardiac function may increase the risk of SUDEP, but these animal data have not been confirmed in humans.

\section{Conclusion}

Dravet syndrome remains one of the most severe epilepsy syndromes of early childhood and is characterized by very high morbidity and mortality. It impacts physical and cognitive function, mental health, bone health and sleep. Anxiety about the risk of death and SUDEP is very high in parents and caregivers. Early diagnosis of Dravet syndrome is important to avoid anti-seizure medications that exacerbate seizures, but there is a major need for new therapies to improve seizure control and long-term outcomes.

Transition from paediatric to adult services is very challenging, and there is a great need to improve this process. ${ }^{53,54}$ It is also likely that Dravet syndrome is underdiagnosed in adults with treatment-resistant epilepsy. It is therefore important to educate colleagues who are caring for adults about this epilepsy syndrome.

\section{DisClosures}

Mary Connolly has the following disclosures: Novartis, site principal investigator, research support; Novartis, speaker, honorarium; UCB, speaker, honorarium. All funds go to the Epilepsy Research and Development Fund (no personal benefit).

\section{REFERENCES}

1. Dravet C. Les epilepsies graves de l'enfant. Vie Med. 1978;8:543-8.

2. Brunklaus A, Ellis R, Reavey E, Forbes GH, Zuberi SM. Prognostic, clinical and demographic features in SCN1A mutation-positive Dravet syndrome. Brain. 2012;135:2329-36. 
3. Bayat A, Hjalgrim H, Moller RS. The incidence of SCN1A-related Dravet syndrome in Denmark is 1:22,000: a population-based study from 2004 to 2009. Epilepsia. 2015;56:36-9.

4. Claes L, Del-Favero J, Ceulemanns B, Lagae L, Van Broeckhoven C, De Jonghe P. De novo mutations in sodium channel SCN1A cause severe myoclonic epilepsy of infancy. Am J Hum Genet. 2001;68:1327-32.

5. Xu X, Zhang Y, Sun H, Liu X, Yang X, Xiong H, et al. Early clinical features and diagnosis of Dravet syndrome in 138 Chinese patients with SCN1A mutations. Brain Dev. 2014;36: 676-81.

6. Hattori J, Ouchida M, Ono J, Miyake S, Maniwa S, Mimaki N, et al. A screening test for the prediction of Dravet syndrome before one year of age. Epilepsia. 2008;49:626-33.

7. Bureau M, Dalla Bernardina B. Electroencephalographic characteristics of Dravet syndrome. Epilepsia. 2011;52(Suppl 2):13-23.

8. Genton P, Velizarova R, Dravet C. Dravet syndrome: the long-term outcome. Epilepsia. 2011;52(Suppl 2):44-9.

9. Caraballo RH, Fejerman N. Dravet syndrome: a study of 53 patients. Epilepsy Res. 2006;70(Suppl 1):S231-8.

10. Takayama R, Fujiwara T, Shigematsu H, Imai K, Takahashi Y, Yamakawa $\mathrm{K}$, et al. Long-term course of Dravet syndrome: a study from an epilepsy center in Japan. Epilepsia. 2014;55: 528-38.

11. Specchio N, Balestri M, Trivisano M, Japaridze N, Striano P, Carotenuto A, et al. Electroencephalographic features in Dravet syndrome: five-year follow-up study in 22 patients. J Child Neurol. 2012;27:439-44.

12. Korff C, Laux L, Kelley K, Goldstein J, Koh S, Nordli D Jr. Dravet syndrome (severe myoclonic epilepsy in infancy): a retrospective study of 16 patients. J Child Neurol. 2007;22:185-94.

13. Kim SH, Nordli DR Jr, Berg AT, Koh S, Laux L. Ictal ontogeny in Dravet syndrome. Clin Neurophysiol. 2015;126:446-55.

14. Nabbout R, Desguerre I, Sabbagh S, Depienne C, Plouin P, Dulac O, et al. An unexpected EEG course in Dravet syndrome. Epilepsy Res. 2008;81:90-5.

15. Escayg A, MacDonald BT, Meisler MH, Baulac S, Huberfeld G, An-Gourfinkel I, et al. Mutations of SCN1A encoding a neuronal sodium channel in two families with GEFS+2. Nat Genet. 2000;24:343-5.

16. Singh R, Andermann E, Whitehouse WP, Harvey AS, Keene DL, Seni MH, et al. Severe myoclonic epilepsy of infancy: extended spectrum of GEFS+? Epilepsia. 2001;42(7):837-44.

17. Depienne C, Trouillard O, Saint-Martin C, Gourfinkel-An I, Bouteiller D, Carpentier W, et al. Spectrum of SCN1A gene mutations associated with Dravet syndrome: analysis of 333 patients. J Med Genet. 2009;46:183-91.

18. Nabbout R, Gennaro E, Dalla Bernardina B, Dulac O, Madia F, Bertini E, et al. Spectrum of SCN1A mutations in severe myoclonic epilepsy of infancy. Neurology. 2003;60:1961-7.

19. Wallace RH, Hodgson BL, Grinton BE, Gardiner RM, Robinson R, Rodriguez-Casero $\mathrm{V}$, et al. Sodium channel alpha1-subunit in severe myoclonic epilepsy of infancy and infantile spasms. Neurology. 2003;61:765-9.

20. Marini C, Mei D, Temudo T, Ferrari AR, Buti D, Dravet C, et al. Idiopathic epilepsies with seizures precipitated by fever and SCN1A abnormalities. Epilepsia. 2007;48:1678-85

21. Marini C, Scheffer IE, Nabbout R, Suls A, De Jonghe P, Zara F, et al. The genetics of Dravet syndrome. Epilepsia. 2011;52(Suppl 2): 24-9.

22. Hirose S, Scheffer IE, Marini C, De Jonghe P, Andermann E, Goldman AM, et al. SCN1A testing for epilepsy: application in clinical practice. Epilepsia. 2013;54:946-52.

23. Martin MS, Tang B, Ta N, Escayg A. Characterization of $5^{\prime}$ untranslated regions of the voltage-gated sodium channels SCN1A, SCN2A, and SCN3A and identification of cis-conserved noncoding sequences. Genomics. 2007;90:225-35.

24. Ohmori I, Ouchida M, Kobayashi K, Jitsumori Y, Mori A, Michiue $\mathrm{H}$, et al. CACNA1A variants may modify the epileptic phenotype of Dravet syndrome. Neurobiol Dis. 2013;50: 209-17.

25. Mulley JC, Hodgson B, McMahon JM, Iona X, Bellows S, Mullen SA, et al. Role of the sodium channel SCN9A in genetic epilepsy with febrile seizures plus and Dravet syndrome. Epilepsia. 2013;54:122-6.

26. Singh NA, Pappasa C, Dahle EJ, Claes LR, Pruess TH, De Jonghe P, et al. A role of SCN9A in human epilepsies, as a cause of febrile seizures and as a potential modifier of Dravet syndrome. PLoS Genet. 2009;5(9):e1000649.

27. Suls A, Jaehn JA, Kecskés A, Weber Y, Weckhuysen S, Craiu DC, et al. De novo loss-of-function mutations in CHD2 cause a fever-sensitive myoclonic epileptic encephalopathy sharing features with Dravet syndrome. Am J Hum Genet. 2013;93: 967-75.

28. Carvill GL, Weckhuysen S, McMahon JM, Hartmann C, Møller RS, Hjalgrim H, et al. GABRA1 and STXBP1: novel genetic causes of Dravet syndrome. Neurology. 2014;82:1245-53.

29. Depienne C, Bouteiller D, Keren B, Cheuret E, Poirier K, Trouillard O, et al. Sporadic infantile epileptic encephalopathy caused by mutations in PCDH19 resembles Dravet syndrome but mainly affects females. PLoS Genet. 2009;5(2):e1000381.

30. Cheah CS, Yu FH, Westenbroek RE, Kalume FK, Oakley JC, Potter GB, et al. Specific deletion of NaV1.1 sodium channels in inhibitory interneurons causes seizures and premature death in a mouse model of Dravet syndrome. Proc Natl Acad Sci U S A. 2012;109:14646-51

31. Yamakawa K. Molecular and cellular basis: insights from experimental models of Dravet syndrome. Epilepsia. 2011;52(Suppl 2): $70-1$.

32. Liu Y, Lopez-Santiago LF, Yuan Y, Jones JM, Zhang H, O'Malley $\mathrm{HA}$, et al. Dravet syndrome patient-derived neurons suggest a novel epilepsy mechanism. Ann Neurol. 2013;74:128-39.

33. Chopra R, Isom LL. Untangling the Dravet syndrome seizure network: the changing face of a rare genetic epilepsy. Epilepsy Curr. 2014;14:86-9.

34. Barba C, Parrini E, Coras R, Galuppi A, Craiu D, Kluger G, et al. Co-occurring malformations of cortical development and SCN1A gene mutations. Epilepsia. 2014;55:1009-19.

35. Gaily E, Anttonen AK, Valanne L, Liukkonen E, Träskelin AL, Polvi A, et al. Dravet syndrome: new potential genetic modifiers, imaging abnormalities, and ictal findings. Epilepsia. 2013;54:1577-85.

36. Pérez A, García-Pentón L, Canales-Rodríguez EJ, Lerma-Usabiaga G, Iturria-Medina Y, Román FJ, et al. Brain morphometry of Dravet syndrome. Epilepsy Res. 2014;108:1326-34.

37. Dravet C, Bureau M, Dalla Bernardina B, Guerrini R. Severe myoclonic epilepsy in infancy (Dravet syndrome) 30 years later. Epilepsia. 2011;52(Suppl 2):1-2.

38. Chieffo D, Ricci D, Baranello G, Martinelli D, Veredice C, Lettori D, et al. Early development in Dravet syndrome: visual function impairment precedes cognitive decline. Epilepsy Res. 2011;93: 73-9.

39. Chieffo D, Battaglia D, Lettori D, Del Re M, Brogna C, Dravet C, et al. Neuropsychological development in children with Dravet syndrome. Epilepsy Res. 2011;95:86-93.

40. Villeneuve N, Laguitton V, Viellard M, Lépine A, Chabrol B, Dravet C, et al. Cognitive and adaptive evaluation of 21 consecutive patients with Dravet syndrome. Epilepsy Behav. 2014;31:143-8.

41. Nabbout R, Chemaly N, Chipaux M, Barcia G, Bouis C, Dubouch C, et al. Encephalopathy in children with Dravet syndrome is not a pure consequence of epilepsy. Orphanet J Rare Dis. 2013;8:176.

42. Akiyama M, Kobayashi K, Yoshinaga H, Ohtsuka Y. A long-term follow-up study of Dravet syndrome up to adulthood. Epilepsia. 2010;51:1043-52.

43. Catarino CB, Liu JY, Liagkouras I, Gibbons VS, Labrum RW, Ellis R, et al. Dravet syndrome as epileptic encephalopathy: evidence from long-term course and neuropathology. Brain. 2011:134:2982-3010.

44. Han S, Tai C, Westenbroek RE, Yu FH, Cheah CS, Potter GB, et al. Autistic-like behaviour in Scn1at/- mice and rescue by enhanced GABA-mediated neurotransmission. Nature. 2012;489:385-90.

45. Bender AC, Natola H, Ndong C, Holmes GL, Scott RC, LenckSantini PP. Focal Scn1a knockdown induces cognitive impairment without seizures. Neurobiol Dis. 2013;54:297-307.

46. Rodda JM, Scheffer IE, McMahon JM, Berkovic SF, Graham HK. Progressive gait deterioration in adolescents with Dravet syndrome. Arch Neurol. 2012;69:873-8. 
47. Fasano A, Borlot F, Lang AE, Andrade DM. Antecollis and levodopa-responsive parkinsonism are late features of Dravet syndrome. Neurology. 2014;82:2250-1.

48. Dhamija R, Erickson MK, St Louis EK, Wirrell E, Kotagal S. Sleep abnormalities in children with Dravet syndrome. Pediatr Neurol. 2014;50:474-8.

49. Skluzacek JV, Watts KP, Parsy O, Wical B, Camfield P. Dravet syndrome and parent associations: the IDEA League experience with comorbid conditions, mortality, management, adaptation, and grief. Epilepsia. 2011;52(Suppl 2):95-101.

50. Kalume F, Westenbroek RE, Cheah CS, Yu FH, Oakley JC, Scheuer $\mathrm{T}$, et al. Sudden unexpected death in a mouse model of Dravet syndrome. J Clin Invest. 2013;123:1798-808.
51. Cheah CS, Westenbroek RE, Roden WH, Kalume F, Oakley JC, Jansen LA, et al. Correlations in timing of sodium channel expression, epilepsy, and sudden death in Dravet syndrome. Channels (Austin). 2013;7:468-72.

52. Auerbach DS, Jones J, Clawson BC, Offord J, Lenk GM, Ogiwara I, et al. Altered cardiac electrophysiology and SUDEP in a model of Dravet syndrome. PLoS One. 2013;8(10):e77843.

53. Ceulemans B. Overall management of patients with Dravet syndrome. Dev Med Child Neurol. 2011;53(Suppl 2):19-23.

54. Kuchenbuch M, Chemaly N, Chiron C, Dulac O, Nabbout R. Transition and transfer from paediatric to adult health care in epilepsy: a family survey on Dravet syndrome. Epilepsy Behav. 2013;29:161-5. 